\title{
Doença do Refluxo Gastroesofágico: uma revisão de literatura
}

\section{Gastroesophageal Reflux Disease: a review of literature}

Pedro Lopes Fraga ${ }^{1}$

Fábio dos Santos Cosso Martins ${ }^{2}$

Artigo

Original

Original

Paper

\section{Palavras-chave:}

Refluxo

Sintomas

Fatores de risco

\section{Resumo:}

O refluxo gastroesofágico (RGE) é definido como o retorno passivo do conteúdo gástrico para o esôfago, independentemente de sua etiologia. Tal fenômeno pode ocorrer em circunstâncias fisiológicas ou patológicas e em qualquer indivíduo, seja criança ou adulto. Quando não está associado a doenças ou complicações, é denominado RGE fisiológico. O RGE patológico, ou doença do refluxo gastroesofágico (DRGE), possui prognóstico mais grave, além de abordagens diagnóstica e terapêutica diferentes. A DRGE cursa com os sintomas típicos (pirose e regurgitação), mas também pode se apresentar com as manifestações atípicas (dor torácica, sintomas respiratórios e otorrinolaringológicos). Em função dessas características, o primeiro passo para o diagnóstico adequado da DRGE é o conhecimento do conceito atual da afecção, dos diversos fatores de risco e das suas várias formas de apresentação clínica.

\section{Abstract}

Gastroesophageal reflux (GER) is defined as the passive return of stomach contents into the esophagus, regardless of its etiology. This phenomenon can occur in physiological or pathological conditions and in any individual, whether child or adult. When not associated with diseases or complications, is called physiological GER. The pathologic GERD, or gastroesophageal reflux disease (GERD), has more serious prognosis, and different approaches to diagnosis and therapy. GERD presents with typical symptoms (heartburn and regurgitation), but also may present with atypical manifestations (chest pain, respiratory symptoms and ENT). Due to these characteristics, the first step to a proper diagnosis of GERD is the knowledge of the current concept of the condition of the various risk factors and their various forms of clinical presentation.
Key words:

Reflux

Symptons

Risk factors
Recebido em $12 / 2011$

Aprovado em $03 / 2012$ 


\section{Introdução}

A doença do refluxo gastroesofágico (DRGE) foi definida pelo Consenso Brasileiro da DRGE como "uma afecção crônica decorrente do fluxo retrógrado de parte do conteúdo gastroduodenal para o esôfago e/ou órgãos adjacentes a ele, acarretando um espectro variável de sintomas e/ou sinais esofagianos e/ou extra-esofagianos, associados ou não a lesões teciduais" (CORSI et al., 2007).

A DRGE pode ser classificada em doença do refluxo erosiva (DRE), pelo encontro de erosões ou evidências de suas complicações na mucosa esofagiana, na presença de sintomas típicos e doença do refluxo não erosiva (DRNE) quando existem os mesmos sintomas, porém sem as lesões acima referidas, ao exame endoscópico. É importante ressaltar que cerca de $50 \%-70 \%$ dos pacientes que procuram auxílio médico têm DRNE, também denominados de pacientes endoscopicamente negativos (AGUERO et al., 2007).

O objetivo desta revisão é analisar os principais sintomas típicos e atípicos da DRGE, no intuito de esclarecer dúvidas sobre aspectos relacionados ao diagnóstico, quais os principais fatores de risco e as possíveis formas de tratamento.

Para a revisão de literatura proposta, foram levantados os mais recentes artigos sobre o tema nas bases de dados Scielo, PubMedMedline e capítulos de livros disponíveis na biblioteca do UniFOA.

\section{Revisão de Literatura}

\subsection{Diferenciação entre refluxo gastro- esofágico fisiológico e patológico}

O refluxo gastroesofágico (RGE) é definido como o retorno passivo do conteúdo gástrico para o esôfago, independentemente de sua etiologia (MAGALHÃES et al., 2009). Tal fenômeno pode ocorrer em circunstâncias fisiológicas ou patológicas e em qualquer indivíduo, seja criança ou adulto. Quando não está associado a doenças ou complicações, é denominado RGE fisiológico. O RGE patológico, ou doença do refluxo gastroesofágico (DRGE), possui prognóstico mais grave, além de abordagens diagnóstica e terapêutica diferentes (JUNG, 2001).

\subsection{Causas e fatores de risco da DRGE}

A causa da DRGE é multifatorial e depende da barreira antirrefluxo (esfíncter esofagiano inferior e diafragma crural), da depuração esofagiana (ação da gravidade, peristalse e salivação), da resistência da mucosa esofágica e/ou do esvaziamento e pressão intragástricos (BICCAS et al., 2009).

$\mathrm{O}$ esfíncter esofagiano inferior (EEI) é composto de músculo liso e está sob controle não colinérgico e não adrenérgico. Ele é mantido em estado de contração constante, principalmente devido à atividade miogênica extrínseca, mas seu tônus de repouso é afetado por vários fatores neurais e hormonais. $\mathrm{O}$ tônus do esfíncter aumenta em resposta às pressões intra-abdominais aumentadas e durante as contrações gástricas (ANDREOLLO; COELHO-NETO; LOPES, 2010).

A idéia de que a obesidade possa ser uma das causas da DRGE é plausível do ponta de vista fisiopatológico. $\mathrm{O}$ excesso de peso tem sido associado a aumento da pressão intra-abdominal, o que por sua vez, aumenta o gradiente de pressão gastroesofágico, a pressão intragástrica e a chance de se desenvolver hérnia hiatal (BICCAS et al., 2009).

Outro fator sugerido é o possível papel da dieta no aparecimento ou agravamento da DRGE nos obesos, que frequentemente abusam da quantidade e qualidade dos alimentos. Refeição rica em gorduras leva à diminuição da pressão basal do esfíncter esofagiano inferior, aumento na freqüência dos relaxamentos transitórios e retardo do esvaziamento do estômago. Se a ingestão for volumosa ou feita de forma muito rápida, ocorrerá distensão do fundo gástrico, aumentando o refluxo pós-prandial (SHAH; KATZ; URIBE, 2005).

Em suma, vários fatores contribuem para tornar o refluxo patológico: número excessivo de episódios de refluxo, depuração esofágica prolongada ou deficiente, menor resistência da mucosa ao conteúdo refluído, ou pela interação do refluxo ácido com co-fatores dietéticos, comportamentais e emocionais (RAPÔSO et al., 2010). A tabela 1 mostra alguns fatores de risco para a DRGE. 


\begin{tabular}{|l|l|l|l|}
\hline 1) & Índice de massa corpórea $\geq 25$ & 5) & Pequeno espaço de tempo entre o jantar e o deitar \\
\hline 2) & Tabagismo & 6) & Trabalhar em posição inclinada \\
\hline 3) & Consumo freqüente de álcool & 7) & Consumo excessivo de alimentos \\
\hline 4) & Estresse e fadiga & 8) & Hérnia de hiato \\
\hline
\end{tabular}

Tabela 1: Resumo dos fatores de risco para a DRGE. Fonte: Tabela feia pelo autor com base na referência KIM et al., 2008.

\subsection{Sintomas típicos, atípicos e condições associadas à DRGE}

Os sintomas clássicos da DRGE são a pirose e a regurgitação, intitulados de sintomas típicos. A pirose consiste na sensação de queimação retrosternal que se irradia do osso esterno à base do pescoço. A regurgitação significa o retorno do conteúdo ácido ou alimentos para a cavidade oral (RAPÔSO et al., 2010). Existem também as manifestações atípicas como a dor torácica, sintomas respiratórios e otorrinolaringológicos, os dois últimos considerados manifestações supra-esofágicas, uma vez que são provocadas pelo efeito do conteúdo gástrico refluído em regiões que ultrapassam o esôfago (AGUERO et al., 2007).

Os sintomas relacionados à doença do refluxo gastroesofágico (DRGE) são extremamente comuns, visto que aproximadamente $20 \%$ dos adultos apresentam pirose e/ou regurgitação pelo menos uma vez por semana e 40\%, mensalmente (GURSK et al., 2006). Se forem consideradas as manifestações extra-esofágicas estima-se que a real prevalência de refluxo patológico possa estar subestimada. A denominação sintomas atípicos serve de sinônimo para os sintomas extra-esofágicos relacionados à DRGE. Consequentemente, um caso de DRGE pode apresentar sinais e sintomas como faringite, dor de ouvido, erosão do esmalte dental, laringite, bronquite, tosse crônica, asma e pneumonia por aspiração (GOLDMAN; AUSIELLO, 2009).

A hérnia hiatal ressurgiu nos últimos anos como importante fator patogênico na DRGE, estando associada a maior exposição ácida esofagiana e sempre presente nas formas mais graves e complicadas da doença. $\mathrm{O}$ mecanismo pelo qual a hérnia hiatal se associa à DRGE mais grave estaria relacionado a maior alteração na função esfincteriana (aumento dos relaxamentos transitórios do esfíncter inferior do esôfago (EIE), à promoção do refluxo ácido e, principalmente, à redução da depuração esofágica observadas sobretudo em hérnias volumosas e não redutíveis (ABRAHÃO et al., 2006).

Embora raramente a DRGE cause morte, essa doença reduz a qualidade de vida e tem um preocupante percentual de morbidade $(10 \%$ a $15 \%)$ (GOLDMAN; AUSIELLO, 2009) por causa de ulceração, sangramento, constrição, esôfago de Barret (substituição do epitélio escamoso lesionado pelo refluxo no esôfago distal por um epitélio colunar especializado metaplásico do tipo intestinal) e adenocarcinoma. Além disso, a DRGE pode ocorrer como conseqüência de outras condições, como síndrome de Zollinger-Ellison (síndrome clínica causada por um tumor endócrino secretor de gastrina), diabetes mellitus, intubação nasogástrica e gravidez (GOLDMAN; AUSIELLO, 2009).

\subsection{Aspectos relacionados ao diagnósti- co da DRGE}

O diagnóstico de DRGE é suspeitado a partir de anamnese e exame físico criteriosos. $\mathrm{Na}$ criança, as queixas mais comuns são dor abdominal, altamente sugestiva se associada às refeições, regurgitações, vômitos freqüentes ou intermitentes, queimação retroesternal, faringodinia matinal, saciedade precoce e raramente disfagia. Irritabilidade e choro freqüente durante a ingestão de alimentos, bem como ingestão diminuída de alimentos, dificuldade em ganhar peso, anemia e fraqueza são indicativos de DRGE e merecem investigação mais detalhada (GUIMARÃES; MARGUET; CAMARGOS, 2006).

O primeiro passo para o diagnóstico adequado da DRGE é o conhecimento do conceito atual da afecção e das suas várias formas de apresentação clínica. Apesar dos sintomas clínicos típicos (pirose e regurgitação) sugeri-

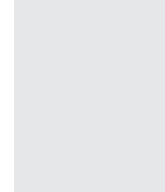

.


rem a presença da afecção, vale salientar que outras doenças (como úlcera péptica, gastrites e eventualmente neoplasias) podem cursar com um deles. Contudo, quando tais queixas co-existem, a possibilidade do paciente ter DRGE é superior a 90\% (NASI; MORAESFILHO; CECCONELLO, 2006).

O diagnóstico clínico da DRGE é bastante sensível, pois a maioria dos pacientes apresenta os sintomas clássicos da doença. Todavia, outros sintomas podem ser referidos pelos refluidores, tais como rouquidão, pigarro, tosse crônica, crises de asma e precordialgias, classificados como atípicos. Tais sintomas podem se apresentar associados aos anteriores ou isoladamente (CORRÊA; LERCO; HENRY, 2008).

É notório salientar que a investigação da DRGE, no período neonatal, reveste-se de importância significativa, já que esta entidade pode ocasionar sintomas graves em recém-nascidos (RNs), sobretudo nos prematuros, tais como crises de apneia, bradicardia, cianose e vômitos. A DRGE também tem sido associada a complicações tais como pneumonias por aspiração e apneias com aparente risco de vida, prolongando de maneira significativa a internação destes RNs (ALVARES; TORRE; MEZZACAPPA, 2011).

O primeiro exame a ser solicitado na suspeita de DRGE é a endoscopia digestiva alta (EDA), pela qual avalia-se a mucosa esofágica, a presença de hérnia hiatal e investiga-se outras afecções do tubo digestivo superior. O avanço tecnológico dos exames endoscópicos permitiu que o diagnóstico da DRGE fosse além dos doentes com hérnia hiatal. Apesar deste avanço, muitos doentes não apresentam esofagite erosiva, úlcera ou esôfago de Barrett (EB). Esses doentes são definidos como "endoscopia negativa" ou portadores de doença do refluxo não erosiva. Os métodos de avaliação funcional do esôfago esclarecem estes casos e aumentam o espectro de apresentação desta complexa afecção (CORSI et al., 2007).

\subsection{Tratamentos farmacológicos e não farmacológicos para a DRGE}

O tratamento clínico visa o alívio dos sintomas, a cicatrização das lesões e a prevenção de recidivas e complicações. Do ponto de vista prático, objetiva-se reduzir o potencial agressivo do conteúdo gástrico, minimizando a agressão representada pelo ácido clorídrico do suco gástrico. Pode-se classificar a abordagem terapêutica em medidas comportamentais e farmacológicas, que deverão ser implementadas simultaneamente (NASI; MORAESFILHO; CECCONELLO, 2006). As medidas comportamentais para se evitar a exacerbação do refluxo patológico estão indicadas a todos os pacientes com suspeita de DRGE e sintomas atípicos. Estas medidas estão apresentadas na tabela 2 (GURSKI et al., 2006).

Elevação da cabeceira da cama $(15 \mathrm{~cm})$.

Moderar a ingestão dos seguintes alimentos, na dependência da correlação com os sintomas: gordurosos, cítricos, café, bebidas alcoólicas, bebidas gasosas, menta, hortelã, produtos de tomate, chocolate.

Cuidados especiais para medicamentos potencialmente "de risco": anticolinérgicos, teofilina, antidepressivos tricíclicos, bloqueadores de canais de cálcio, agonistas beta adrenérgicos, alendronato.

Evitar deitar-se nas duas horas após as refeições.

Evitar refeições copiosas.

Redução drástica ou cessação do fumo.

Redução do peso corporal (emagrecimento). 
O tratamento do RGE pode ser medicamentoso através de bloqueadores de hidrogênio, agentes procinéticos e inibidores de bomba de próton. Os bloqueadores de hidrogênio atuam na diminuição do ácido gástrico. Os agentes procinéticos aceleram o esvaziamento do esôfago e estômago, produzindo maior pressão no esfíncter inferior e os inibidores de bomba de próton promovem a inibição da produção do ácido gástrico. A intervenção cirúrgica também pode ser necessária em pacientes com sintomas crônicos e que não respondem satisfatoriamente ao tratamento medicamentoso (CIELO et al., 2011).

Os bloqueadores $\mathrm{H}_{2}$ se ligam de modo reversível aos receptores $\mathrm{H}_{2}$ da célula parietal, inibindo a resposta secretória ácida desses receptores. Apresentam efetividade comprovada e são usados por milhões de pessoas no mundo. A eficácia clínica da droga depende da inibição gástrica desejada e de aspectos inerentes a essa inibição. Essa classe de drogas é mais eficiente em inibir a secreção ácida basal, particularmente a secreção ácida noturna. No mercado estão disponíveis cimetidina, ranitidina, famotidina e nizatidina. Dentre essas drogas, a ranitidina é a mais prescrita no nosso meio (GUIMARÃES; MARGUET; CAMARGOS, 2006).

No manejo do RGE e da DRGE, procinéticos têm sido usados há muitos anos. São drogas que estimulam a motilidade do aparelho digestivo por ação direta sobre o músculo liso entérico ou por interação com os neurônios do sistema nervoso entérico (MAGALHÃES et al., 2009).

Os inibidores da bomba de prótons (IBP) são substâncias que inibem seletiva e completamente a bomba de prótons $\mathrm{H}^{+} \mathrm{K}^{+}$ATPase (bomba de prótons) na membrana da célula parietal. A secreção gástrica ácida é suprimida em resposta a todos os agentes estimulantes até que novas moléculas da bomba sejam sintetizadas. Estas drogas reduzem a secreção de ácido clorídrico em cerca de 90\% (contra $65 \%$ dos inibidores $\mathrm{H}_{2}$ ). Também melhoram a proteção da barreira mucosa pelo aumento da produção de muco e do fluxo sanguíneo na mucosa gástrica (PEDROSO; OLIVEIRA, 2007).

Os IBP em uso clínico no mundo são omeprazol, lansoprazom, pantoprazol, rabeprazol e esomeprazol. Embora semelhantes em sua estrutura, os IBP apresentam diferenças em relação ao metabolismo. Os IBP, principalmente omeprazol, são metabolizados em graus variados pelo sistema enzimático hepático P450 (GUIMARÃES; MARGUET; CAMARGOS, 2006). Estudo baseado em revisão sistemática de literatura (VAN et al., 2005) avaliando a eficiência de IBP e antagonistas dos receptores $\mathrm{H}_{2}$ em adultos com sintomas típicos da DRGE sem esofagite ao estudo endoscópico, conclui que os agentes anti-secretores são eficientes no controle da queixa clínica desses pacientes e que os IBP apresentam resultados melhores que os antagonistas dos receptores $\mathrm{H}_{2}$.

\section{Discussão e Considerações Finais}

O quadro clínico de doentes com DRGE divide-se em três categorias clínicas principais. A primeira é composta por sintomas típicos mais comuns, como azia e regurgitação que pioram após as refeições quando o paciente se deita e/ou se curva. A segunda, relaciona-se com as complicações da DRGE (disfagia leve na ausência de qualquer estreitamento; odinofagia; hematêmese e melena). A terceira categoria inclui disfagia orofaríngea, asma, dor torácica recorrente e faringite. A halitose, a salivação amarga, o gosto ácido na boca e problemas dentários devem ser considerados inespecíficos e não obrigatoriamente associados com o refluxo (ANDREOLLO; LOPES; COELHO-NETO, 2010).

De acordo com Vicente et al. (2009), podem ocorrer alterações da DRGE relacionadas à nutrição, à mucosa esofágica, à função respiratória ou ao aparecimento de sintomas neurocomportamentais. Em extenso estudo populacional realizado em Pelotas, RS, Oliveira et al. (2005), observaram que a DRGE apresenta maior prevalência entre as mulheres, em geral associada a eventos estressantes negativos e mal estar psicológico.

Segundo Mendes-Filho et al. (2010), existe correlação entre os distúrbios motores esofágicos e a doença do refluxo gastroesofágico, e, portanto, o tratamento cirúrgico- que é responsável por corrigir a acalásia e o refluxo gastroesofágico ao mesmo tempo- pode ser indicado em casos selecionados.

Conforme as idéias supracitadas e baseadas em Corsi et al. (2007), o primeiro exame a ser solicitado na suspeita de DRGE é a endoscopia digestiva alta, mas segundo Ratier et al. (2011) apesar de a endoscopia digestiva 
alta ser o método mais confiável para detectar a esofagite causada por DRGE, sua ausência não exclui o diagnóstico da mesma. Dessa forma, o exame clínico e a avaliação dos sintomas presentes são de suma importância para a elaboração de hipóteses diagnósticas consistentes e para a eficácia dos tratamentos.

\section{Conclusões}

A doença do refluxo gastroesofágico cursa com os sintomas típicos (pirose e regurgitação), mas também pode se apresentar com as

\section{Referências Bibliográficas}

1. ABRAHÃO, L. J. et al. Relação entre o tamanho de hérnia hiatal e tempo de exposição ácida esofágica nas doenças do refluxo erosivae não-erosiva. Arquivo de Gastroenterologia., São Paulo, v. 43, n. 1, Mar. 2006.

2. Aguero, G. C. et al. Prevalência de queixas supra-esofágicas em pacientes com doenças do refluxo erosiva e não-erosiva. Arquivo de Gastroenterologia., São Paulo, v. 44, n. 1, Mar. 2007.

3. ALVARES, B. R.; TORRE, O. H. D.; MEZZACAPPA, M. A. Sensibilidade da seriografia do esôfago, estômago e duodeno para o diagnóstico de doença do refluxo gastroesofágico em recém-nascidos prematuros. Radiologia Brasileira., São Paulo, v. 44, n. 4, Aug. 2011.

4. ANDREOLLO, N. A.; LOPES, L. R.; COELHO-NETO, J. S. Doença do refluxo gastroesofágico: qual a eficácia dos exames no diagnóstico? ABCD, arq. bras. cir. dig., São Paulo, v. 23, n. 1, Mar. 2010.

5. BICCAS, B. N. et al. Maior prevalência de obesidade na doença do refluxo gastroesofagiano erosiva. Arq. Gastroenterol., São Paulo, v. 46, n. 1, Mar. 2009.

6. CIELO, C. A. et al. Refluxo laringofaríngeo e bulimia nervosa: alterações vocais e laríngeas. Rev. CEFAC., São Paulo, v. 13, n. 2, Apr. 2011. manifestações atípicas (dor torácica, sintomas respiratórios e otorrinolaringológicos). Em função dessas características, o primeiro passo para o diagnóstico adequado da DRGE é o conhecimento do conceito atual da afecção, dos diversos fatores de risco e das suas várias formas de apresentação clínica.

O tratamento desta patologia deve ser medicamentoso (através do uso de fármacos específicos) e não medicamentoso (através de medidas comportamentais). Até mesmo a intervenção cirúrgica pode ser necessária em pacientes com sintomas crônicos e que não respondem satisfatoriamente ao tratamento medicamentoso.

7. CORRÊA, M. C. C. S. F.; LERCO, M. M.; HENRY, M. A. C. A. Estudo de alterações na cavidade oral em pacientes com doença do refluxo gastroesofágico. Arq. Gastroenterol., São Paulo, v. 45, n. 2, June 2008.

8. CORSI, P. R. et al. Factors related to the presence of reflux in patients with typical symptoms of gastroesophageal reflux disease (GERD). Rev. Assoc. Med. Bras., São Paulo, v. 53, n. 2, Apr. 2007.

9. GOldman, L.; AUSIEllo, D. Cecil Medicina. Rio de Janeiro: Elsevier, 2009.

10. Guimarães, E. V.; MARguet, C.; CAMARGOS, P. A. M. Tratamento da doença do refluxo gastroesofágico. J. Pediatr. (Rio J.)., Porto Alegre, v. 82, n. 5, Nov. 2006.

11. GURSKI, R. R. et al. Manifestações extra-esofágicas da doença do refluxo gastroesofágico. J. bras. pneumol., São Paulo, v. 32, n. 2, Apr. 2006.

12. JUNG, A.D. Gastroesophageal reflux in infants and children. Am Farm Physician., v.64 p.1853-60, 2001.

13. KIM, N. et al. The prevalence of and risk factors for erosive o esophagitis and non-erosive reflux disease: a nationwide multicentre prospective study in Korea. Aliment Pharmacol Ther., v.27 p.173-85, 2008. 
14. MAGALHÃES, P. V. S. et al. Revisão sistemática e metanálise do uso de procinéticos no refluxo gastroesofágico e na doença do refluxo gastroesofágico em Pediatria. Revista paulista de Pediatria., São Paulo, v. 27, n. 3, Sept. 2009.

15. MENDES-FILHO, A. M. et al. Evolução motora incomum na doença do refluxo gastroesofágico. ABCD, arq. bras. cir. dig., São Paulo, v. 23, n. 3, Sept. 2010.

16. NASI, A.; MORAES-FILHO, J. P. P.; CECCONELlo, I. Doença do refluxo gastroesofágico: revisão ampliada. Arq. Gastroenterol., São Paulo, v. 43, n. 4, Dec. 2006.

17. OLIVEIRA, S. S. et al. Prevalência e fatores associados à doença do refluxo gastroesofágico. Arq. Gastroenterol., São Paulo, v. 42, n. 2, June 2005.

18. PEDROSO, E. R. P.; OLIVEIRA, R. G. Blackbook Clínica Médica., Belo Horizonte: Blackbook Editora, 2007.

19. RAPÔSO, N. M. L.; CRUZ, M. C. F. N.; LOPES, F. F. A erosão dentária como uma lesão oral predominante na doença do refluxo gastroesofágico. Revista brasileira de odontologia., Rio de Janeiro, v. 67, n. 2, p.152-6, jul./dez. 2010.
20. RATIER, J. C. A.; PIZZICHINI, E.; PIZZICHINI, M. Doença do refluxo gastroesofágico e hiperresponsividade das vias aéreas: coexistência além da chance? J. bras. pneumol., São Paulo, v. 37, n. 5, Oct. 2011.

21. SHAH, A.; URIBE, J.; KATZ, P.O. Gastroesophageal reflux disease and obesity. Gastroenterol Clin North Am., v.34 p.35-43, 2005.

22. VAN, P. B.; NUMANAS, M.E.; BONIS, P.A.; LAU, J. Short-term treatment with protonpump inhibitors, H2receptor antagonists and prokinetics for gastro-esophageal reflux diseaselike symptoms and endoscopy negative reflux disease. (Cochrane Review). In: The Cochrane Library, Issue 2, 2005.

23. VICENTE, A. M. B. et al. Evolução clínica e endoscópica após fundoplicatura para tratamento da doença do refluxo gastroesofágico. Arq. Gastroenterol., São Paulo, v. 46, n. 2, June 2009.

Endereço para Correspondência:

Pedro Lopes Fraga

pedrolfraga@hotmail.com

Centro Universitário de Volta Redonda - UniFOA

Campus Olezio Galotti

Av. Paulo Erlei Alves Abrantes, ${ }^{\circ} 1325$

Três Poços - Volta Redonda - RJ

CEP: 27240-000 